\title{
Recent developments in Marshall-Olkin distributions
}

\author{
Jiju Gillariose ${ }^{1}$, Lishamol Tomy ${ }^{2}$, Christophe Chesneau ${ }^{3, *}$, Manju Jose $^{4}$ \\ ${ }^{1}$ Department of Statistics, St. Thomas College, Pala, Kerala, India \\ ${ }^{2}$ Department of Statistics, Deva Matha College, Kuravilangad, Kerala, India \\ ${ }^{3}$ Laboratoire de Mathématiques Nicolas Oresme, Université de Caen, Campus II, Caen, France \\ ${ }^{4}$ Department of Statistics, Alphonsa College, Pala, Kerala, India
}

(Received: 28 August 2020. Received in revised form: 3 November 2020. Accepted: 18 December 2020. Published online: 31 December 2020.)

(c) 2020 the authors. This is an open access article under the CC BY (International 4.0) license (www.creativecommons.org/licenses/by/4.0/).

\begin{abstract}
Over the past two decades, a significant part of the statistical literature has been devoted to offer distinct univariate distributions belonging to the Marshall-Olkin family of distributions. It is because this family enjoys attractive statistical properties, providing consistently better fit than the other generalized distributions with the same parental models, as well as wider applications. In this article, we provide a brief review of recent developments in Marshall-Olkin type distributions.
\end{abstract}

Keywords: Hazard rate function; Marshall-Olkin distribution; probability mass function.

2020 Mathematics Subject Classification: 60E05, 62E15.

\section{Introduction}

Introducing parameters in well-known families of distributions has become a topic of high importance in academic research due to their compulsive properties. Indeed, in general, one-parameter families of distributions are not sufficient to handle various real contexts, and this fact motivates the construction of new distributions with more than one parameter. So, new parameters are introduced for expanding the classical distributions to obtain more flexible new families of distributions. In addition, this parameter induction is helpful in improving the goodness-of-fit of the related statistical models.

Several authors have developed diverse parameter plug-in techniques, with applications in various factual contexts. Among them, Marshall and Olkin [37] introduced a general method for obtaining more flexible distributions by adding a new parameter to an existing family of distributions. The obtained family is named as the Marshall-Olkin (MO) family of distributions. Several researchers showed that distributions obtained from the MO scheme are enough to analyze data presenting high or moderate skewness. Moreover, the basic motivations for using the MO family in practice are the following:

- To make the kurtosis more flexible compared to the parental distribution.

- To acquire a pliant skewness for symmetrical parental distributions.

- To construct heavy-tailed distributions.

- To develop distributions with symmetric, left-skewed, right-skewed or reversed-J shape.

- To specify special distributions with all types of hazard rate function (HRF).

- To furnish better fits than other extended models under the same parental model, with at least an equal number of parameters.

As previously mentioned, in comparison to the parental distribution, the MO family of distributions includes one more parameter denoted by $\eta$. More technical details are now presented. Let us consider a parental continuous distribution with a cumulative density function (CDF) denoted by $F(x)$ and survival function (SF) denoted by $\bar{F}(x)$, and a tuning parameter $\eta>0$. Then, we can obtain a new family of CDFs based on the MO scheme, called univariate MO family of distributions, and its CDF is given by the following formula:

$$
G(x ; \eta)=\frac{F(x)}{\eta+(1-\eta) F(x)} ; x \in \mathbb{R}
$$


or equivalently:

$$
G(x ; \eta)=\frac{F(x)}{1-(1-\eta) \bar{F}(x)} ; x \in \mathbb{R}
$$

and the corresponding SF is obtained as

$$
\bar{G}(x ; \eta)=\frac{\eta \bar{F}(x)}{1-(1-\eta) \bar{F}(x)} ; x \in \mathbb{R}
$$

If $\eta=1$ then the parental CDF appears; we have $G(x ; \eta)=F(x)$. Otherwise, the following dominance results hold: for $\eta \in(0,1)$, we have $F(x) \leq G(x ; \eta)$, and the reverse ineqality holds for $\eta \geq 1$.

Globally, owing to $\eta, G(x ; \eta)$ has more flexible capabilities to the parental distribution. This aspect is also reflected in the definitions of the corresponding probability density function (PDF) and HRF. By denoting $f(x)$ and $r(x)$ the PDF and $\mathrm{HRF}$ of the parental distribution, respectively, the PDF of the MO family of distributions is given by

$$
g(x ; \eta)=\frac{\eta f(x)}{(\eta+(1-\eta) F(x))^{2}} ; x \in \mathbb{R}
$$

and its corresponding HRF is given by

$$
r(x ; \eta)=\frac{r(x)}{\eta+(1-\eta) F(x)} ; x \in \mathbb{R} .
$$

In particular, we see that $r(x) \leq r(x ; \eta)$ for $\eta \in(0,1)$, and the reverse inequality holds for $\eta \geq 1$. In addition, the quantile function $(\mathrm{QF})$ of the $\mathrm{MO}$ family is quite manageable; it is defined by

$$
Q(y ; \eta)=Q_{F}\left(\frac{\eta y}{1-(1-\eta) y}\right) ; y \in(0,1),
$$

where $Q_{F}(y)$ denotes the $\mathrm{QF}$ of the parental distribution.

The MO family is based on the following theory. Let $X_{1}, X_{2}, \ldots$ be a sequence of independent and identically distributed random variables with SF $\bar{F}(x)$ and $T$ be a geometric random variable independent of $X_{1}, X_{2}, \ldots$ with probability mass function (PMF) $P(T=t)=\eta(1-\eta)^{t-1}, t=1,2, \ldots, \eta \in(0,1)$, then $U_{T}=\min \left(X_{1}, \ldots, X_{T}\right)$ has the SF $\bar{G}(x ; \eta)$ in Equation (1). Also, if $\eta>1$ and if $T$ follows the geometric distribution with PMF

$$
P(T=t)=\eta^{-1}\left(1-\eta^{-1}\right)^{t-1},
$$

then $W_{T}=\max \left(X_{1}, \ldots, X_{T}\right)$ has the $\mathrm{SF} \bar{G}(x ; \eta)$ in Equation (1).

The bivariate version of the MO family of distributions is also popular. In this case, if $(X, Y)$ is a random vector with joint CDF denoted by $F(x, y)$ and joint SF denoted by $\bar{F}(x, y)$, then the joint CDF is given by

$$
G(x, y ; \eta)=\frac{F(x, y)}{\eta+(1-\eta) F(x, y)} ;(x, y) \in \mathbb{R}^{2}
$$

or equivalently:

$$
G(x, y ; \eta)=\frac{F(x, y)}{1-(1-\eta) \bar{F}(x, y)} ;(x, y) \in \mathbb{R}^{2}
$$

constitutes the MO bivariate family of distributions. Here again, the new parameter $\eta$ results in added flexibility of the parental distribution and influences the reliability properties. Similarly to the univariate case, the corresponding SF is obtained as

$$
\bar{G}(x, y ; \eta)=\frac{\eta \bar{F}(x, y)}{1-(1-\eta) \bar{F}(x, y)} ;(x, y) \in \mathbb{R}^{2} .
$$

The corresponding PDF and HRF can be expressed in a similar manner.

Since the introduction of the MO family, an extensive work has been done including different interpretations, generalizations, inferential methods, discrete cases and extensions to bivariate and multivariate cases; details can be found in $[49,50,52]$. In this paper, we give a short review on the recent developments of the MO family of distributions.

The organization of the rest of the paper is as follows: Section 2 deals with different generalizations of the MO family of distributions. Section 3 describes the MO types distributions including some of their relevant properties. Finally, concluding remarks are given in Section 4. 


\section{A survey of generalizations of the MO family of distribution}

The literature on the MO family is dispersed across many different scientific journals. In this section, we focus only on the latest works. Recently, several new flexible distributions based on the MO family have been introduced in the literature. We mention some of them: MO family of heavy-tailed distributions [20], MO additive Weibull distribution [2], MO Kumaraswamy distribution [21], MO generalized Erlang-truncated exponential distribution [40], MO inverted Kumaraswamy distribution [51], MO alpha power family of distributions [39], MO logistic-exponential distribution [35], MO power inverse exponential distribution [11], MO kappa distribution [27], MO power Lomax distribution [22], MO lengthbiased exponential distribution [53] and Weibull MO Lindley distribution [3]. In addition to this, some new discrete models have been appeared in the literature from existing continuous MO distributions. For instance, we refer the papers [45], [28] and [29], where the discrete generalized MO versions of uniform, exponential and Weibull distributions, respectively, are investigated. Moreover, a new discrete MO family of distributions is introduced in [23].

Recently, various properties of the MO family have been discussed by a number of authors. The prediction problem of the MO exponential distribution based on the Bayesian framework under progressive Type-II censoring was studied in [15]. A new class of defective distributions based on the MO family has been introduced in [44]. In [13], it was proved that the MO family characteristics are preserved under marginalization. Constant-partially accelerated life tests for the MO exponential series system with dependent masked data are described in [19]. The authors of [10] introduced the Type-I progressive interval censoring schemes of the MO bivariate Weibull (MOBW) distribution and they suggested the confidence intervals depending on percentile bootstrap of the unknown parameters. A dependent competing risks model using the MOBW model was developed in [10].

The problem of acceptance sampling based on truncated life tests is another area of research. In this regard, the authors of [7] proposed acceptance sampling plans from truncated life tests using MO Esscher transformed Laplace. The Bayesian techniques to examine visualization of prognosis of women with breast cancer using three distributions, namely the MO exponential distribution, MO exponentiated exponential distribution and MO with exponentiated extension distribution, has been discussed in [1].

In addition, several attempts have been made to define generalizations of the MO family which give great flexibility in modelling data in practice. Some contributed works on generalized MO family of distributions are enlisted in Table 1.

\section{A survey of latest developments of the MO type distributions}

This section provides some very recent discussions on the MO type distributions. The multivariate distribution has become one of the important classical tools to specify the variety of dependence structures. The MO distributions are widely used in high-dimensional models. In particular, the MO scheme seems to be one of the best schemes to analyze various dependence structures. The basic properties of the MO bivariate exponential distribution and its correlation structure have been studied in [34]. The MOBW distribution is an important distribution of MO type distributions, which consists of singular and absolute continuous parts. The MOBW distribution also has a correlation control parameter, which may be used to express the relationship of dependent competing risks. Ozkut and Bayramoglu [41] discussed the problem of the MO type distributions with effect of shock magnitude. Feizjavadian and Hashemi [18] analyzed the problem of dependent competing risks in the presence of the progressive hybrid censoring by using the MOBW distribution, and gave two illustrative examples based on the practical data sets.

Stability of systems is an important issue in the field of natural sciences. In stochastic system, stability represents insensitivity or low sensitivity of their output characteristics to the shapes of some input distributions. Some articles have been devoted to these issues. For example, the reliability function of the two-component reliability system with MO failure model for its elements was discussed in [33]. The MO type shock model in coherent systems was considered in [16]. The reliability, mean residual life and inactivity time of a coherent system subjected to the MO type shocks were discussed in [12]. Moreover, run shock and MO models were discussed in [42].

\section{Conclusion}

The MO family of distributions has a nice physical interpretation, so it can be used as an alternative to well-known distributions in the literature. Induction of the new parameter increases the flexibility of the parental model. This scheme is found to be useful for modelling distinct practical contexts in recent years. In addition, the MO family can be applied in different areas, like reliability theory, hydrology, medicine, meteorology, survival analysis, acceptance sampling, record 
Table 1: Various generalized MO families of distributions

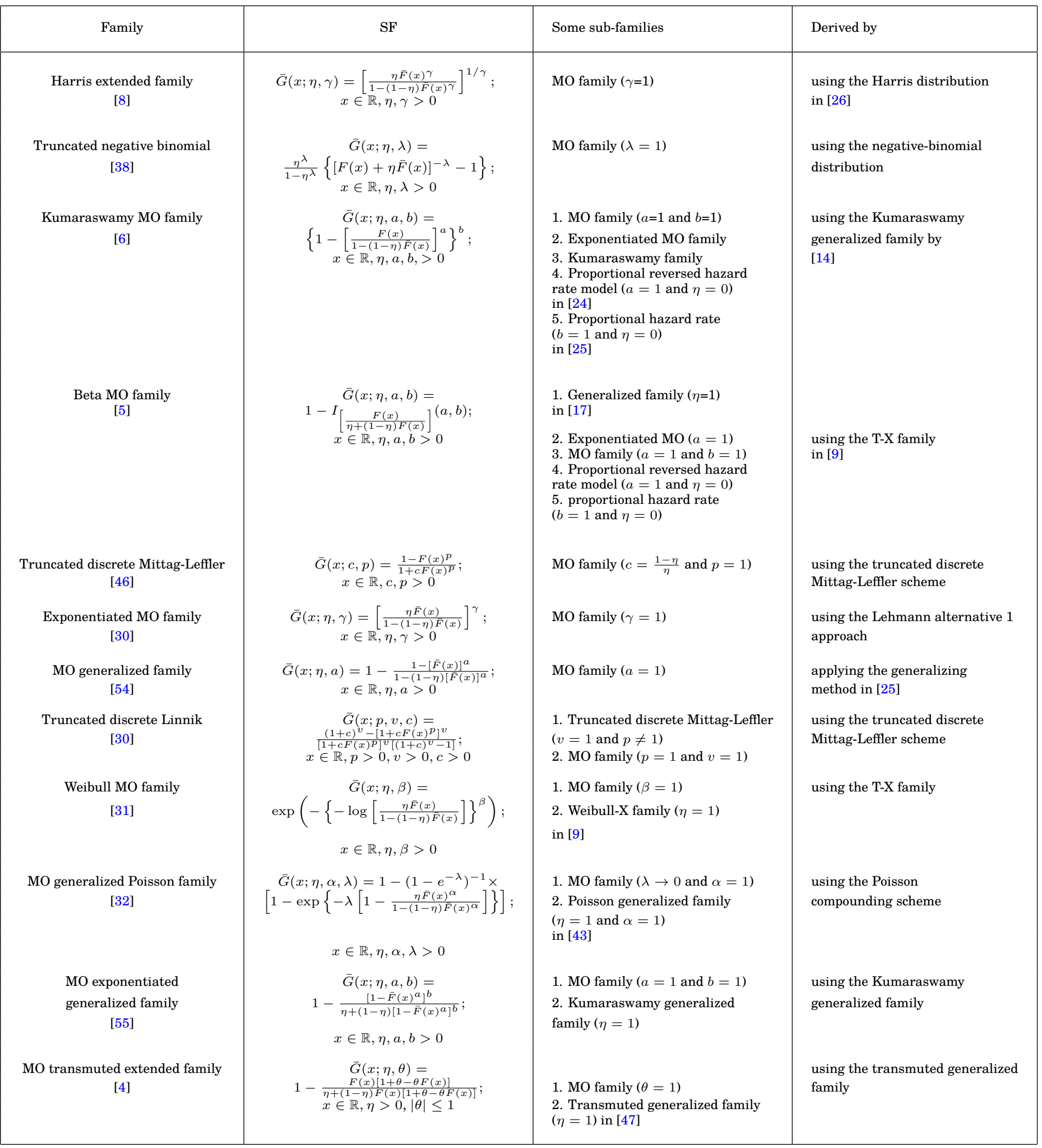

value theory, time series modelling and engineering. The recent developments of the MO distributions are discussed in this paper.

\section{Acknowledgments}

The authors would like to thank the two anonymous referees for corrections and the suggestions of recent references on the topic. The first author is thankful to the Department of Science and Technology (DST), Government of India, for funding. 


\section{References}

[1] M. H. Abu-Jarad, A. A Khan, M. A. Khaleel, E. S. A. AbuJarad, A. H. AbuJarad, Bayesian reliability analysis of marshall and olkin model, Ann. Data Sci. 7 (2020) 461-489.

[2] A. Z. Afify, G. M. Cordeiro, H. M. Yousof, A. Saboor, E. M. M. Ortega, The Marshall-Olkin additive Weibull distribution with variable shapes for the hazard rate, Hacettepe J. Math. Stat. 47 (2016) 365-381.

[3] A. Z. Afify, M. Nassar, G. M. Cordeiro, D. Kumar, The Weibull Marshall-Olkin Lindley distribution: properties and estimation, J. Taibah Univ. Sci. $14(2020)$ 192-204.

[4] A. Z. Afify, H. M. Yousof, M. Alizadeh, I. Ghosh, S. Ray, G. Ozel, The Marshall-Olkin Transmuted-G family of distributions, Stoch. Qual. Control 35 (2020) 79-96.

[5] M. Alizadeh, G. M. Cordeiro, E. Brito, C. Demétrio, The beta Marshall-Olkin family of distributions, J. Stat. Distrib. Appl. 2 (2015) Art\# 4.

[6] M. Alizadeh, H. M. Tahir, G. M. Cordeiro, M. Zubair, G. G. Hamedani, The Kumaraswamy Marshal-Olkin family of distributions, J. Egypt. Math. Soc. 23 (2015) 546-557.

[7] A. I. Al-Omari, M. Aslam, A. D. Al-Nasser, Acceptance sampling plans from truncated life tests using Marshall-Olkin Esscher transformed Laplace distribution, J. Reliab. Stat. Stud. 11 (2018) 103-115.

[8] E. Aly, E. Benkherouf, A new family of distributions based on probability generating functions, Sankhya B 73 (2011) 70-80.

[9] A. Alzaatreh, C. Lee, F. Famoye, A new method for generating families of continuous distributions, METRON 71 (2013) 63-79.

[10] X. Bai, Y. Shi, B. Liu, Q. Fu, Statistical inference of Marshall Olkin bivariate Weibull distribution with three shocks based on progressive interval censored data. Commun. Stat. Simul. Comput. 48 (2019) 637-654.

[11] A. M. Basheer, Marshall-Olkin alpha power inverse exponential distribution: properties and applications, Ann. Data Sci. 20 (2019) 1-3.

[12] I. Bayramoglu, M. Ozkut, Mean residual life and inactivity time of a coherent system subjected to Marshall-Olkin type shocks, J. Comput. Appl. Math. 298 (2016) 190-200.

[13] D. Brigo, J. Mai, M. Scherer, Markov multi-variate survival indicators for default simulation as a new characterization of the Marshall-Olkin law, Stat. Prob. Lett. 114 (2017) 60-66.

[14] G. M. Cordeiro, M. Castro, A new family of generalized distributions, J. Stat. Comput. Simul. 81 (2011) $883-893$.

[15] S. Dey, M. Nassar, R. K. Maurya, Y. M. Tripathi, Estimation and prediction of Marshall-Olkin extended exponential distribution under progressively type-II censored data, J. Stat. Comput. Simul. 88 (2018) 2287-2308.

[16] F. Durante, S. Girard, G. Mazo, Marshall-Olkin type copulas generated by a global shock, J. Comput. Appl. Math. 296 (2016) 638-648.

[17] N. Eugene, C. Lee, F. Famoye, Beta-normal distribution and its applications, Commun. Stat. Theor. Meth. 31 (2002) 497-512.

[18] S. H. Feizjavadian, R. Hashemi, Analysis of dependent competing risks in the presence of progressive hybrid censoring using Marshall-Olkin bivariate Weibull distribution, Comput. Stat. Data Anal. 82 (2015) 19-34.

[19] Q. Fu, Y. Shi, Constant-partially accelerated life tests for Marshall-Olkin exponential series system with dependent masked data, Commun. Stat. Simul. Comput. 46 (2017) 7879-7893.

[20] V. J. Garciá, E. Gómez-Déniz, F. J. Vázquez-Polo, Marshall-Olkin family of heavy-tailed distributions which includes the lognormal one, Commun. Stat. Theor. Meth. 45 (2016) 2023-2044.

[21] R. George, S. Thobias, Marshall-Olkin Kumaraswamy distribution, Int. Math. Forum 12 (2017) 47-69.

[22] J. Gillariose, L. Tomy, The Marshall-Olkin extended power Lomax distribution with applications, Pak. J. Stat. Oper. Res. 3 (2020) 331-41.

[23] J. Gillariose, L. Tomy, F. Jamal, C. Chesneau, A new discrete Marshall-Olkin family of distributions, Preprint.

[24] R. C. Gupta, R. D. Gupta, Proportional reversed hazard rate model and its applications, J. Stat. Plan. Inference 137 (2007) $3525-3536$.

[25] R. C. Gupta, P. L. Gupta, R. D. Gupta, Modeling failure time data by Lehman alternatives, Commun. Stat. Theor. Meth. 27 (1998) 887-904.

[26] T. E. Harris, Branching processes, Ann. Math. Stat. 19 (1948) 474-494.

[27] M. Javed, T. Nawaz, M. Irfan, The Marshall-Olkin kappa distribution: properties and applications, J. King Saud Univ. Sci. 31 (2019) 684-691.

[28] K. Jayakumar, K. K. Sankaran, A discrete generalization of Marshall-Olkin scheme and its application to geometric distribution, J. Kerala Stat. Assoc. 28 (2017) 1-21.

[29] K. Jayakumar, K. K. Sankaran, A generalization of discrete Weibull distribution, Commun. Stat. Simul. Comput. 47 (2017) $6064-6078$.

[30] K. Jayakumar, K. K. Sankaran, Discrete Linnik Weibull distribution, Commun. Stat. Simul. Comput. 48 (2019) 3092-3117.

[31] M. C. Korkmaz, G. M. Cordeiro, H. M. Yousof. The Weibull Marshall-Olkin family: regression model and applications to censored data, Commun. Stat. Theor. Meth. 48 (2018) 4171-4194.

[32] M. C. Korkmaz, H. M. Yousof, G. G. Hamedani, M. M. Ali, The Marshall-Olkin generalized G Poisson family of distributions, Pak. J. Stat. 34 (2018) 251-267.

[33] D. Kozyrev, N. Kolev, V. Rykov, Reliability function of renewable system under Marshall-Olkin failure model, Reliab. Theory Appl. 13 (2018) $39-46$.

[34] G. D. Lin, C. D. Lai, K. Govindaraju, Correlation structure of the Marshall-Olkin bivariate exponential distribution, Stat. Methodol. 29 (2016) 1-9.

[35] M. Mansoor, M. H. Tahir, G. M. Cordeiro, S. B. Provost, A. Alzaatreh, The Marshall-Olkin logistic-exponential distribution, Commun. Stat. Theor. Meth. 48 (2019) 220-234.

[36] A. W. Marshall, I. Olkin, A multivariate exponential distribution, J. Amer. Stat. Assoc. 62 (1967) 30-44.

[37] A. W. Marshall, I. Olkin, A new method for adding a parameter to a family of distributions with application to the exponential and Weibull families, Biometrica 84 (1997) 641-652.

[38] S. Nadarajah, K. Jayakumar, M. M. Ristić. A new family of lifetime models, J. Stat. Comput Simul. 83 (2012) 1-16.

[39] M. Nassar, D. Kumar, S. Dey, G. M. Cordeiro, A. Z. Afify, The Marshall-Olkin alpha power family of distributions with applications, J. Comput. Appl. Math. 351 (2019) 41-53.

[40] I. E. Okorie, A. C. Akpanta, J. Ohakwe, Marshall-Olkin generalized Erlang-truncated exponential distribution: Properties and applications, Cogent Math. Stat. 4 (2017) 73-84.

[41] M. Ozkut, I. Bayramoglu, On Marshall-Olkin type distribution with effect of shock magnitude, J. Comput. Appl. Math. 271 (2014) $150-162$.

[42] M. Ozkut, S. Eryilmaz, Reliability analysis under Marshall-Olkin run shock model, J. Comput. Appl. Math. 349 (2019) 52-59.

[43] M. W. Ramos, P. R. D. Marinho, G. M. Cordeiro, R. V. Silva, G. Hamedani, The Kumaraswamy-G Poisson family of distributions, J. Stat. Theory App. 14 (2015) 222-239.

[44] R. Rocha, S. Nadarajah, V. Tomazella, F. Louzada, A new class of defective models based on the Marshall-Olkin family of distributions for cure rate modelling, Comput. Stat. Data Anal. 107 (2017) 48-63.

[45] E. Sandhya, C.B. Prasanth, Marshall-Olkin discrete uniform distribution, J. Prob. 2014 (2014) Art\# 979312.

[46] K. K Sankaran, K. Jayakumar, A new extended uniform distribution, Int. J. Stat. Distib. Appl. 2 (2016) 35-41.

[47] W. T. Shaw, I. R. Buckley, The alchemy of probability distributions: beyond Gram-Charlier expansions and a skew-kurtotic-normal distribution from a rank transmutation map, ArXiv: 0901.0434 [q-fin.ST] (2007), Preprint.

[48] Y. Shen A. Xu, On the dependent competing risks using Marshall-Olkin bivariate Weibull model: parameter estimation with different methods, Commun. Stat. Theor. Meth. 47 (2018) 5558-5572.

[49] M. H. Tahir, S. Nadarajah, Parameter induction in continuous univariate distributions: Well established G families, An. Acad. Bras. Ciénc. 87 (2015) 539-568.

[50] S. P. Thomas, K. K. Jose, L. Tomy, Marshall-Olkin generalized family of distributions: a retrospect. Res. Rev. J. Stat. 6 (2017) 35-49.

[51] L. Tomy, J. Gillariose, The Marshall-Olkin Ikum distribution, Biom. Biostat. Int. J. 7 (2018) 10-16.

[52] L. Tomy, K. K. Jose, P. T. Sophia, Applications of Marshall-Olkin generalized family of distributions, Res. Rev. J. Stat. 7 (2018) $22-37$.

[53] M. A. Ul-Haq, R. M. Usman, S. Hashmi, A. I. Al-Omeri, The Marshall-Olkin length-biased exponential distribution and its applications, J. King Saud Univ. Sci. 31 (2019) 246-251.

[54] H. M. Yousof, A. Afify, S. Nadarajah, G. Hamedani, G. Aryal, The Marshall-Olkin generalized-G family of distributions with applications, Statistica $\mathbf{7 8}(2018)$ 273-295.

[55] H. M. Yousof, M. Rasekhi, M. Alizadeh, G. G. Hamedani, The Marshall-Olkin exponentiated generalized G family of distributions: properties, applications and characterizations, J. Nonlinear Sci. Appl. 13 (2020) 34-52. 